\title{
Research Paper: \\ Content Validity and Reliability of the Persian Version of School Function Assessment in Children With Cerebral Palsy Aged 7-12 Years
}

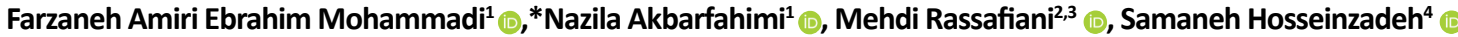

1. Department of Occupational Therapy, University of Social Welfare and Rehabilitation Sciences, Tehran, Iran.

2. Pediatric Neurorehabilitation Research Center, University of Social Welfare and Rehabilitation Sciences, Tehran, Iran.

3. Department of Occupational Therapy, Faculty of Applied Medical Sciences, Kuwait University, Kuwait City, Kuwait.

4. Department of Biostatistics, University of Social Welfare and Rehabilitation Sciences, Tehran, Iran.

\begin{tabular}{|c|c|}
\hline 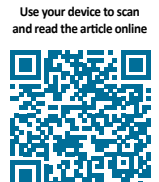 & $\begin{array}{l}\text { Citration Amiri Ebrahim Mohammadi F, Akbarfahimi N, Rassafiani M, Hosseinzadeh S. [Content Validity and Reliability } \\
\text { of the Persian Version of School Function Assessment in Children With Cerebral Palsy Aged 7-12 Years (Persian)]. Archives of } \\
\text { Rehabilitation. 2020; 21(2):138-153. https://doi.org/10.32598/RJ.21.2.543.4 } \\
\text { doi) https://doi.org/10.32598/RJ.21.2.543.4 }\end{array}$ \\
\hline
\end{tabular}

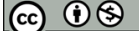

Received: 25 Jan 2019 Accepted: 07 Oct 2019 Available Online: 01 Jul 2020

Keywords:

Cerebral palsy, Schoo function assessment Content validity, Test-retest reliability, Internal consistency

\begin{abstract}
Objective Cerebral Palsy is one of the most common neurodevelopmental disorders in children. Due to the increase in the number of students with Cerebral Palsy (CP) entering ordinary schools and facing problems with independent functioning, there is a need for a valid and comprehensive assessment tool for their successful entry as well as providing a proper treatment plan. The School Function Assessment (SFA), by measuring many school-related functional skills, can identify the strengths and weaknesses affecting the students' independence and participation in school assignments. It has 320 items and three main parts of participation, task support, and activity performance. The test score can be reported in general or based on each part, separately. The purpose of this study is to examine the validity and reliability of the Persian version of the SFA in children with CP.

Materials \& Methods This is a cross-sectional study with methodological design. The study population consists of all children with CP aged 7-12 years in Tehran. Of these, 120 were selected from exceptional public school using a convenience sampling method. The inclusion criteria were: CP diagnosed by a neurologist, age between 7 and 12 years, no other disabilities (e.g. deafness or blindness), and the willingness of children's parents to participate in the study. A demographic form and the Persian SFA were used for data collection through interview. In order to measure the content validity, Content Validity Index (CVI) and Content Validity Ratio (CVR) were calculated according to the standard protocol of International Quality of Life Assessment. In this regard, 8 experts in the field of pediatrics completed the Persian SFA. To determine the test-retest reliability, the questionnaire was completed by the parents of children with a 4-week interval. The internal consistency and test-retest reliability were examined by using Cronbach's alpha coefficient and Intra-class Correlation Coefficient (ICC), respectively. Data was analyzed in SPSS V. 22 software.

Results Participants were 64 boys and 56 girls with CP (mean age $=9.4$ years). The distribution of CP was as follows: $10 \%$ hemiplegic $C P, 27.5 \%$ diplegic $C P, 5.8 \%$ monoplegic $C P$, and $56.7 \%$ quadriplegic $C P$. Eighty hundred-eight percent of respondents were the mothers of students. The CVR and CVI values were in a range of 0.7-1 and 0.87-1, respectively indicating an acceptable validity. All the items had acceptable content validity. The Cronbach's alpha coefficient was obtained 0.95 for participation, $0.95-0.99$ for task support, 0.83-0.99 for activity performance, and 0.92 in total. Moreover, the ICC value was reported 0.90 for participation; $0.84-0.91$ for task support; 0.84-0.97 for activity performance; and 0.89 for the overall test. Conclusion The Persian version of SFA has acceptable content validity and reliability (internal consistency and test-retest) for children with CP. Therefore, it can be applied as a research and clinical tool to assess the school-related performance of these children.
\end{abstract}

\section{* Corresponding Author:}

Nazila Akbarfahimi, PhD.

Address: Department of Occupational Therapy, University of Social Welfare and Rehabilitation Sciences, Tehran, Iran.

Tel: +98 (912) 1753987

E-Mail: fahimi1970@yahoo.com 


\section{English Version}

\section{Introduction}

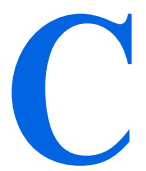

erebral Palsy (CP) is the most common chronic motor disability and a neurological complication in children, resulted from a non-progressive lesion in the developing brain which causes limitation in manual activity, impaired balance, decreased quality of life, and decreased participation in social activities $[1,2]$. One area of participation for children with $\mathrm{CP}$ is attending school. Children with $\mathrm{CP}$ are often difficult to perform the expected functions in schools due to physical, cognitive, and social deficits affecting their ability to actively participate in learning activities and interaction with peers [3].

On the other hand, CP-related problems such as movement planning impairment, learning disorders, IQ and cognitive problems, vision and hearing impairments and difficulty communicating with peers, and emotional and psychosocial disorders, affect their level of participation and performance in school $[2,4]$. Therefore, careful study of the factors affecting the performance of these children requires the use of a comprehensive and accurate tool. School Function Assessment (SFA) was first developed in the United States in 1998 by Coster et al., to measure a wide range of school-related functional skills.

The test consists of three main parts: 1: to examine the student's level of participation in different school activity settings; 2: To examine the support currently provided to the student when s/he performs school-related functional tasks; and 3: To examine the student's ability to perform specific functional tasks, including physical and cognitive dimensions. These three parts identify strengths and limitations affecting child participation in a wide range of schoolrelated tasks $[5,6]$. Unlike traditional tests (e.g. Peabody developmental motor scale and motor skill test) that are designed primarily for normal children, SFA can be used in children with a wide range of functional disorders including sensory, behavioral, emotional, cognitive and motor deficits. The only disadvantage of this test is the high number of questions which has been somewhat solved by considering a separate score for each part and the possibility of reporting the results of only one part of the assessment.

In Iran, most of the used assessment tools measure the child's academic skills and cognitive and physical dimensions, including hand function. Unfortunately, there is no exact statistics of assessments used in schools, but it seems that the most common assessment tool for school performance is the academic skills assessment tool. These tools are not able to examine all the problems of these children. Therefore, it seems necessary to have access to a test that, in addition to covering these skills, examines the non-academic school-related performance of SP children with a focus on rehabilitation. The aim of this study is to evaluate the content validity and reliability of the Persian version of the SFA in CP children.

\section{Materials \& Methods}

This is a descriptive/correlational study with cross-sectional design. The study population consists of all children with CP studying in ordinary and exceptional schools in Tehran, Iran during 2016-2017. Of these, 120 were selected using a convenience sampling method. Inclusion criteria were: CP diagnosed by a neurologist, age between 7 and 12 years, CP without concomitant disability (blindness or deafness), and completion of a consent form to participate in the study by parents. All the questionnaires were answered by the students' parents. In case of their unwillingness to continue participation, students were excluded from the study. A demographic form and SFA questionnaire was used. The SFA has 320 items examining participation (7 items), task support (21 items) and activity performance (292 items). Each question is scored numerically on a scale from 1 to 6 , where score 1 indicates extremely limited participation and score 6 shows full participation $[5,6]$.

After obtaining the necessary permissions, to evaluate the reliability and validity of the Persian SFA test, the standard protocol of International Quality Of Life Assessment (IQOLA) and Lawshe method were used. The content validity of the test was assessed by eight experts and therapists in the field of children with $\mathrm{CP}$ in terms of simplicity, relevance, clarity and necessity. Scoring for each item was done according to Lawshe method [7-9]. To evaluate the test- retest reliability of the Persian SFA, we referred to three exceptional schools in Tehran named Soroush, Tavankhahan and Imam Ali, and the centers affiliated to the rehabilitation schools of Tehran University of Medical Sciences. After selecting the eligible students, a day was arranged as a briefing session for the participants in each school or center.

Due to the high number of questions and the possibility of being tired during completion, parents of students were explained so that if desired, the questionnaire can be provided to them for 24 hours to answer the questions or the questions can be read for them by the rater in one or two sessions. After providing the necessary explanations to them, written consent was obtained from the parents and the SFA questionnaire was completed based on the method selected by them. The questionnaires were completed through interview during 90-120 minutes. The en- 
tire interview process took place during the hours that parents were at schools. After four weeks, the SFA form was completed again by the parents of all 120 children and the results of both pretest and posttest stages were recorded. They were analyzed in SPSS V. 22 software.

\section{Results}

Of the 120 students participating in the study, 53\% were boys and $47 \%$ were girls with an mean age of 9.4 years (ranged 7-12 years). The distribution of CP was as follows: $10 \%$ hemiplegic $\mathrm{CP}, 27.5 \%$ diplegic $\mathrm{CP}, 5.8 \%$ monoplegic $\mathrm{CP}$, and $56.7 \%$ quadriplegic $\mathrm{CP}$. Table 1 shows the demographic characteristics of children and their parents. According to the interpretation of the CVI scores, a score above 0.79 is appropriate; a score between 0.70 and 0.79 is questionable and needs to be corrected or revised; and a score less than 0.70 is unacceptable [8]. The results showed that the CVI value was between 0.87 and 1 , which is an acceptable value. Therefore, all parts have good content validity.

According to Lawshe [8], CVR $>0.62$ is required for eight experts to retain the items. The results of this study showed a CVR of 0.7-1, which indicates an acceptable CVR. Cronbach's alpha calculation was used to check the internal consistency of the three parts and ICC was used to check the test-retest reliability, the results of which are shown in Tables 2 and 3. Cronbach's alpha $>0.9$ indicate excellent reliability; 0.8-0.9, good; 0.7-0.8, acceptable; 0.6-0.7, questionable; 0.5-0.6, poor; and $\alpha<0.5$, unacceptable reliability. Regarding ICC values, scores $>0.9$ indicates excellent reliability; 0.75-0.9, good; 0.5 - 0.75 , moderate; and $<0.5$ shows poor reliability [10].

The Cronbach's alpha coefficients, shown in Table 2, show an excellent reliability for the first and second parts and a good-to-excellent reliability for the third part, indicating that the Persian SFA has excellent internal consistency. The ICC values, shown in Table 3, show an excellent reliability for the first part and a good-to-excellent reliability for the second and third parts of the Persian SFA.

\section{Discussion}

Many of the assessment available tools have been developed based on the skills of normal children in ordinary schools. As a result, they are not well able to assess the challenges facing children with CP. This study examined the validity and reliability of SFA-Persian version in schoolaged children with CP. Hwang et al. translated and developed a Chinese version of SFA for cross-cultural use in Taiwan [11]. Due to differences in the linguistics structure between Chinese and English, incomprehensible sentences were seen in the translated version. Therefore, to increase the readability of the translation, a number of items were adapted by giving examples or using synonymous words.

For example, due to the fact that the presence of cafeterias is not common in Taiwanese schools, the item of eating in the cafeteria was replaced by eating in the classroom, and items such as card games and ground activities were removed. In the end, a total of 14 items were matched, 13 were deleted, and 3 were added [11]. In the study by Shojaei et al. in Iran, some of the items were culturally adapted. For example, since the Iranian schools do not have a bath for children in the school, this word was changed to the word "toilet"; the dining area replaced the cafeteria; children's football replaced kickball; Vasati game replaced dodgeball; classroom equipment replaced classroom utensils; and USB flash drives or CDs replaced tape or floppy disks. Moreover, the unit of measurement was changed from 20 feet to 6 meters, and direction was changed from "left to right" to "right to left" [9]. The present study investigates the validity and reliability of this developed Persian version.

The results showed that all items in the Persian version of this test have good content validity for children with cerebral palsy (CVI $>0.7)$. Content validity of the Persian version of this test is appropriate for normal children in Iran, except in 5 items (CVI <0.7) [9]. For children with movement disorders, its content validity was reported to be acceptable except in 23 items (CVI <0.42) [12]. The content validity of the Chinese version of SFA was also suitable for children with CP (CVI >0.7) [13]. Hwang et al. [14] investigated convergent and known-groups validity of SFA on 64 children: 29 in general education without disabilities, 18 with learning disabilities, and 17 with CP.

The convergent validity was assessed by the Wayland Adaptive Behavior Scale (VABS). The results showed a moderate to high relationship (0.5-0.7) between comparable areas of the SFA and VABS. The results of knowngroups validity showed a significant difference in all three parts of SFA between the three groups. There was no significant difference between normal children and those with learning disabilities in participation and physical tasks, but a significant difference in cognitive-behavioral tasks of these children was observed. Their results, moreover, showed that there was a significant difference in both physical and cognitive-behavioral domains between children with $\mathrm{CP}$ and normal children [14]. Our study was performed only to evaluate the content validity of the Persian SFA on 120 children with $\mathrm{CP}$, and a good content validity was obtained.

The ICC for the participation part of the Persian SFA was obtained 0.90; for the second part, it was 0.91 in physical 
Table 1. Demographic characteristics of children and their parents

\begin{tabular}{|c|c|c|}
\hline \multicolumn{2}{|c|}{ Characteristics } & \multirow{2}{*}{$\begin{array}{c}\text { No. (\%) } \\
26(7.21)\end{array}$} \\
\hline & 7 & \\
\hline & 8 & $16(3.13)$ \\
\hline & 9 & $17(2.14)$ \\
\hline \multicolumn{3}{|c|}{ Age of children (y) } \\
\hline & 10 & $22(3.18)$ \\
\hline & 11 & $22(3.18)$ \\
\hline & 12 & $17(2.14)$ \\
\hline & Monoplegia & $7(8.5)$ \\
\hline & Hemiplegia & $12(10)$ \\
\hline \multicolumn{3}{|c|}{ Type of CP } \\
\hline & Diplegia & $33(5.27)$ \\
\hline & Quadriplegia & $68(7.56)$ \\
\hline Total & & $120(100)$ \\
\hline & Girl & $56(7.46)$ \\
\hline \multicolumn{3}{|c|}{ Gender of children } \\
\hline & Boy & $64(3.53)$ \\
\hline Total & & $120(100)$ \\
\hline \multirow{5}{*}{ Education level of } & Junior high school & $39(5.32)$ \\
\hline & High school diploma & $46(3.38)$ \\
\hline & & \\
\hline & Bachelor degree & $25(8.20)$ \\
\hline & $\begin{array}{c}\text { Master degree and } \\
\text { higher }\end{array}$ & $10(3.8)$ \\
\hline Total & & $120(100)$ \\
\hline & Female & $97(8.80)$ \\
\hline \multicolumn{3}{|c|}{ Gender of caregivers } \\
\hline & Male & $23(2.19)$ \\
\hline Total & & $120(100)$ \\
\hline \multirow{5}{*}{ Age of caregiv } & $30-20$ & $38(7.31)$ \\
\hline & $40-30$ & $56(7.46)$ \\
\hline & & \\
\hline & $50-40$ & $18(15)$ \\
\hline & $60-50$ & $8(7.6)$ \\
\hline Total & & $120(100)$ \\
\hline
\end{tabular}


Table 2. Cronbach's alpha values for testing the internal consistency of the Persian SFA

\begin{tabular}{ccc}
\hline SFA Parts & Sub-sections & $\boldsymbol{\alpha}$ \\
\hline Participation & & 0.95 \\
& Physical tasks & 0.99 \\
Task support & Cognitive-behavioral tasks & 0.95 \\
\hline Activity performance & & $0.83-0.99$ \\
Total & 0.92 \\
\hline
\end{tabular}

Archives of

Table 3. ICC results for examining the test-retest reliability of the Persian SFA

\begin{tabular}{ccc}
\hline SFA Parts & Sub-sections & ICC \\
\hline Participation & & 0.90 \\
& Physical tasks & 0.91 \\
Task support & Cognitive-behavioral tasks & 0.84 \\
\hline Activity performance & & $0.84-0.97$ \\
\hline Total & 0.89 & \\
\hline
\end{tabular}

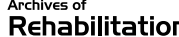

tasks and 0.84 in cognitive-behavioral tasks; and for the third part it was between 0.83 and 0.97 , which indicates its good reliability. The test-retest reliability using ICC for the original version of SFA on children with a wide range of movement disorders (including 6 children with $\mathrm{CP}$ ) for three parts of participation, task support, and activity performance was reported $0.95,0.80-0.97$, and $0.90-0.99$, respectively with a total value ranged $0.82-0.98[5,6]$.

The test-retest reliability of Chinese version of SFA on 14 children with CP aged 6-18 years using ICC was reported 0.83 for participation, $0.78-0.84$ in physical tasks and 0.69 0.82 in cognitive-behavioral tasks related to the second part, and 0.49-0.97 for the third part, and "moderate" in total [13]. The test-retest reliability of the Persian version of the SFA was evaluated by 40 teachers of normal children with an interval of two weeks, which showed that the ICC was in the range of 0.75-0.97 which was good in overall.

Although the ICC value in cognitive-behavioral and physical tasks was in the good range, but perhaps the reason for the low ICC score in these two domains ( 0.84 in maintaining and changing position as a cognitive-behavioral task, and 0.89 in functional communication as a physical task) can be related to large motor involvement (due to the quadriple- gia prevalence of $56.7 \%$ among students) and consequently, low self-confidence in movement and communication.

In assessing the internal consistency of the Persian SFA, Cronbach's alpha coefficient for the participation part was reported 0.95; physical tasks, 0.99; cognitive-behavioral tasks, 0.95; and activity performance, 0.83-0.99; and 0.92, in total (excellent). This is while the internal consistency using the Cronbach's alpha calculation method for the Persian version of SFA on 80 children with various movement disorders is 0.96 (excellent). The results of examining the internal consistency of the original version of SFA during its design and development by Rush Analysis method on 363 children with various mobility impairments reported a Cronbach's alpha of 0.92-0.93 for the participation part, $0.94-0.96$ for task support, $0.93-0.98$ for activity performance, and 0.82-0.92 in total $[5,6]$.

For its Chinese version, Cronbach's alpha coefficient for the participation part was reported 0.92 ; physical tasks, 0.94 and cognitive-behavioral tasks, 0.93 both related to task support section; and activity performance, 0.91-0.97; and 0.92 , in total (excellent) [13]. Although their reported Cronbach's alpha coefficient is at good level for the task support part, but perhaps the reason for its lower score com- 
pared to that reported in our study, especially in safety item (0.84), can be attributed to environmental problems and insufficient environmental facilitators such as educational facilities and equipment in exceptional schools in Tehran.

\section{Conclussion}

Overall, it can be concluded that the Persian version of SFA has good content validity and reliability for children with CP in Iran, and therapists can use it to determine and advance treatment goals to improve the work performance of CP children. The limitation of this study was the length of the interview time, which reduced the parent's willingness to retake the test. Moreover, due to the lack of access to similar articles on the validity and reliability of this test in children with $\mathrm{CP}$, it was not possible to provide more comparisons. Studies are suggested to evaluate the reliability between therapists and caregivers of children with $\mathrm{CP}$ and children with other defects. Furthermore, it seems that structural validity and factor analysis can complement this study.

\section{Ethical Considerations}

\section{Compliance with ethical guidelines}

Ethical considerations were observed in this study. Ethical approval was obtained from the Research Ethics Committee of the University of social welfare and Rehabilitation Sciences (IR: USWR.REC.1396.29).

\section{Funding}

This study was extracted from the MSc. thesis of first author in the Department of Occupational Therapy, University of Social Welfare and Rehabilitation Sciences, Tehran.

\section{Authors' contributions}

Conceptualization: Nazila Akbarfahimi, Mehdi Rassafiani; Methodology: Farzaneh Amiri Ebrahim Mohammadi, Samaneh Hosseinzadeh; Investigation: Farzaneh Amiri Ebrahim Mohammadi; Writing-original draft: Nazila Akbarfahimi, Farzaneh Amiri Ebrahim Mohammadi; Writing-review \& editing: All author

\section{Conflicts of interest}

The authors declared no conflict of interest.

\section{Acknowledgements}

Authors officials of exceptional schools (Soroush, Tavankhahan and Imam Ali) who participated and cooperated in this study. 
This Page Intentionally Left Blank 
مقاله بزوهشى

\title{
بررسى رواييى محتوايى و يايايى نسخه فارسى أزمون عملكرد مدرسه در كودكان فلج مغزى V-Y Iال
}

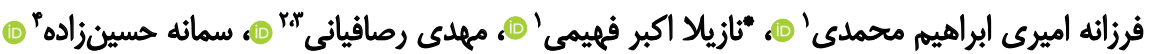

\author{
1. ا.كروه كاردرمانى، دانشعاه علوم بهزيستى و توانبخشى، تهران، ايران.

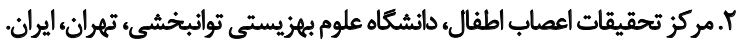

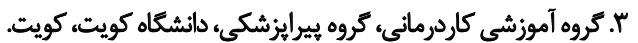

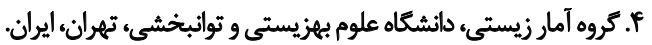

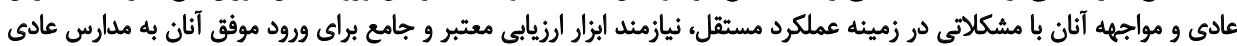

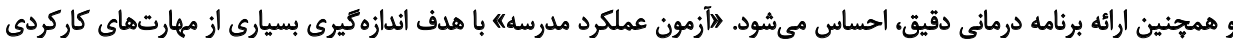

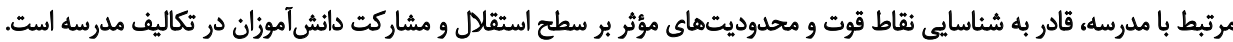

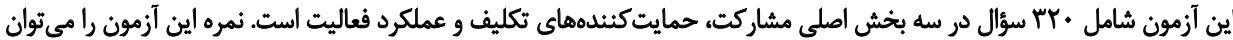

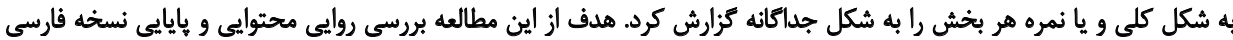

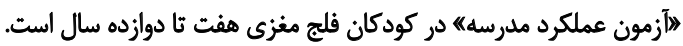

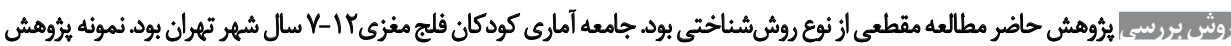

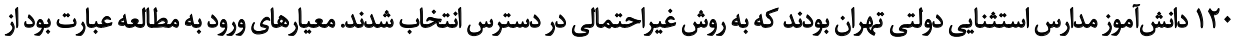

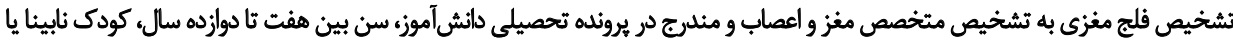

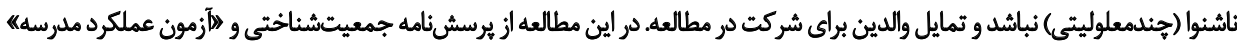

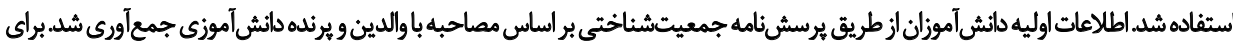

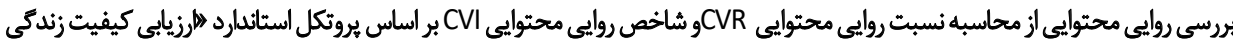

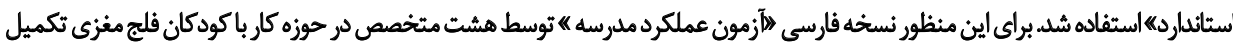

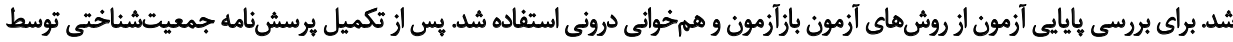

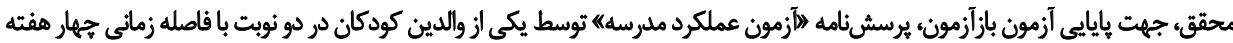

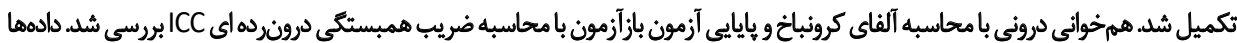

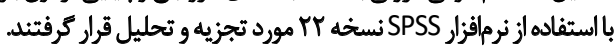

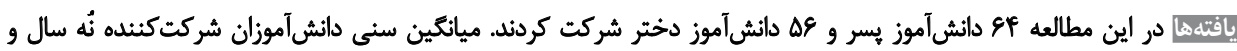

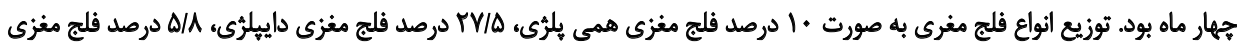

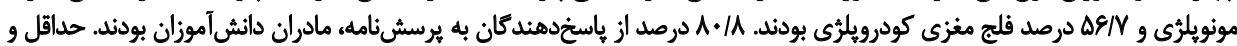

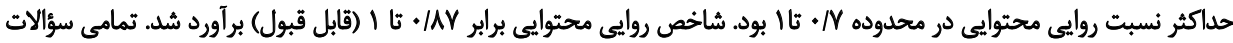

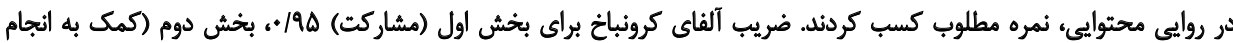

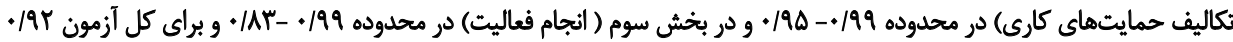

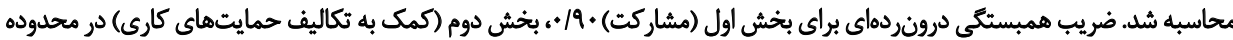

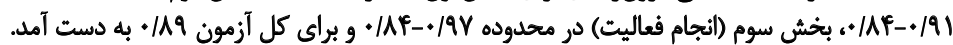

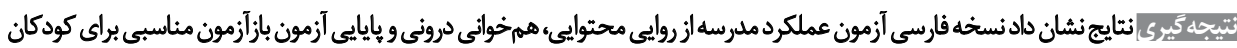

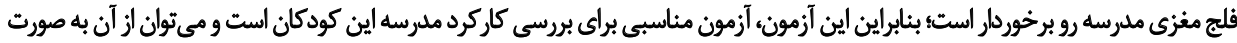

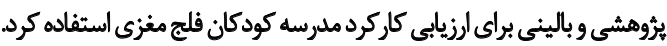


ارزيابي دستخط كودى" [9]، ارزيابى دستخط مينه سوتاه

مقدمه

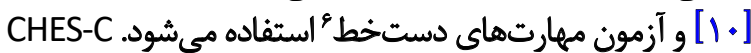

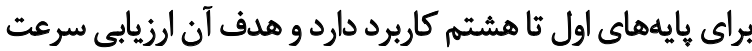

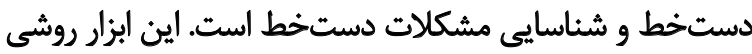

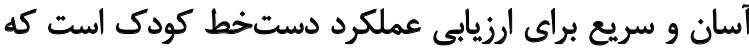

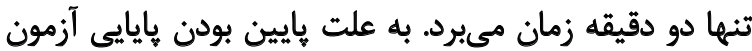

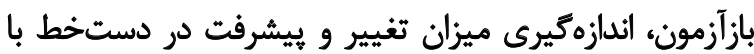

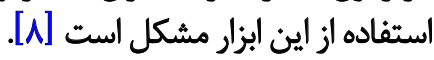
ETCH

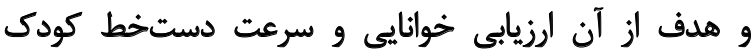

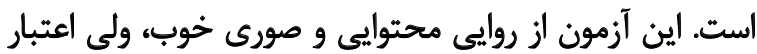

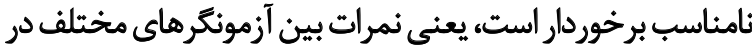

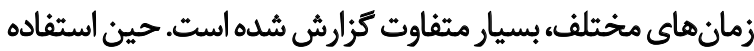

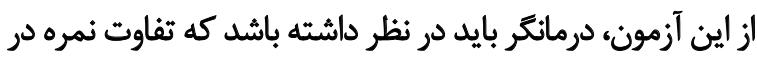

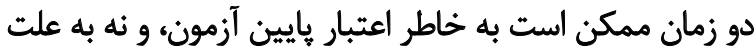

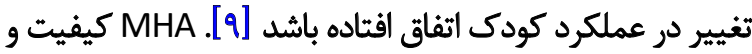

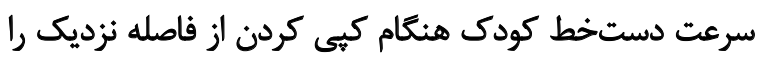

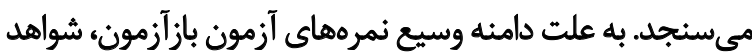

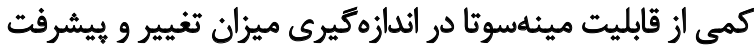
در عملكرد كودكى حمايت مي كنيند [.1].]

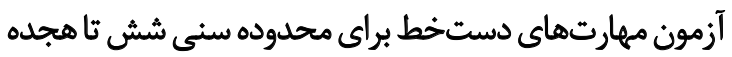

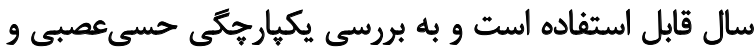

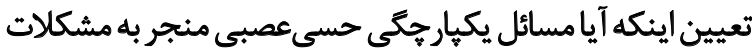

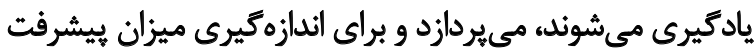

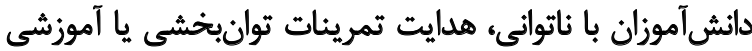

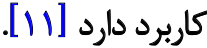

از جمله ابزارهاى ارزيابى رايج شئاختى مقياس هوش وكسلر

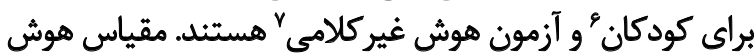
وكسلر براى سنين شش الى شاني انزده سال كاربرد

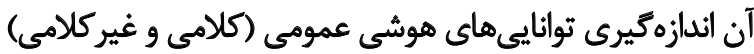

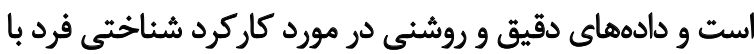

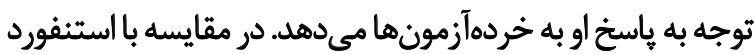

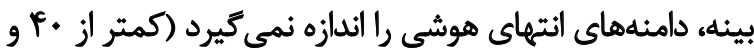

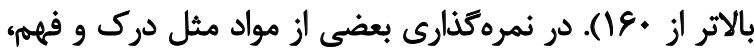

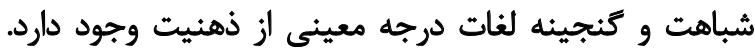

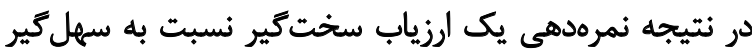

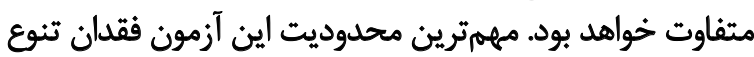

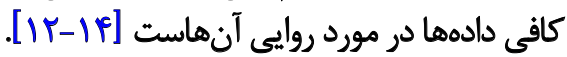

4. Evaluation Tool of Children's Handwriting (ETCH)

5. Minnesota Handwriting Assessment (MHA)

6. Wechsler Intelligence Scale for Children

7. Test of Nonverbal Intelligence

1. Participation and Environment Measure (PEM-C)
2. Children's Assessment Participation And Enjoyment (CAPE)

3. Children's Handwriting Evaluation Scale (CHES-C)

فلج مغزى شايعترين ناتوانى حركتى مزمن و و عارضه

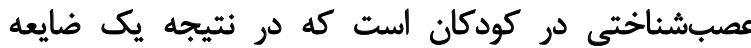

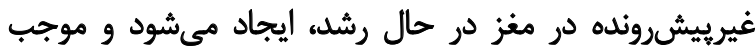

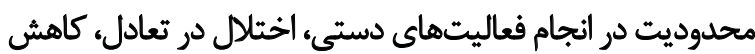

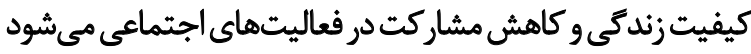

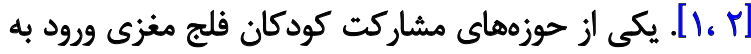

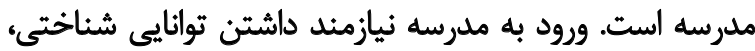

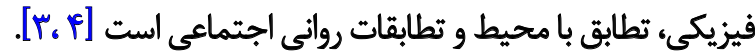
دانش آموزان فلج مغزى اغلب به علت نقايص فيزيكي، شناختى

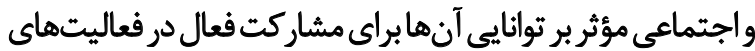

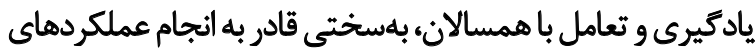

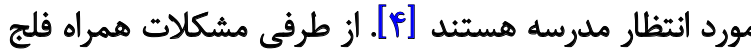

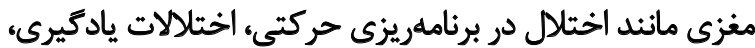

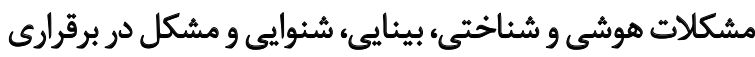

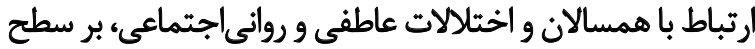

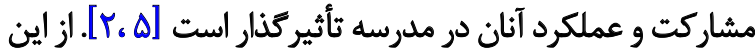

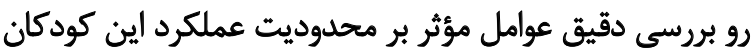
مسثلزم استفاده از ابزارى جامع مؤر ير دقيق است

در حال حاضر در دنيا از آزمونهاى مختلفى براى ارزيابى نوائ نوائي

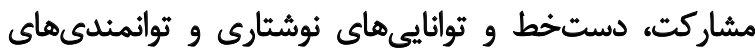

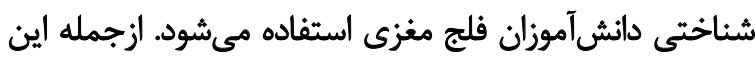

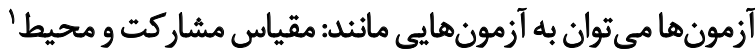
و ارزيابى مشاركت و لذت كودى ازمّا اشاره كرد.

PEM-C

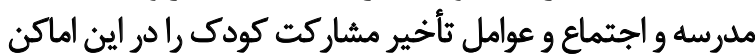

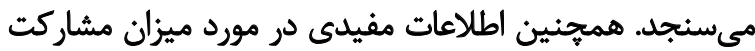

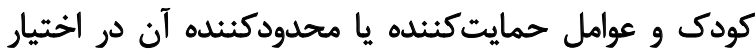

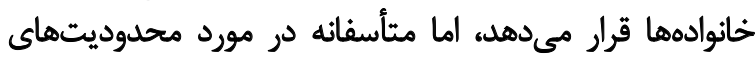

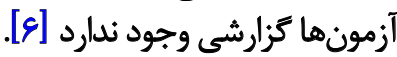
CAPE

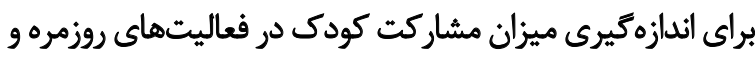

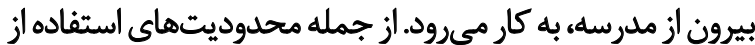

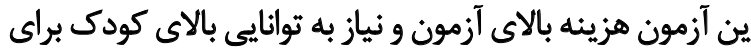

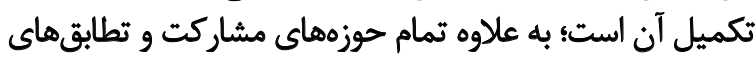

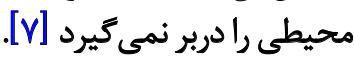

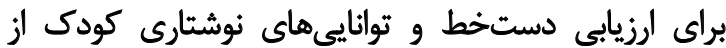

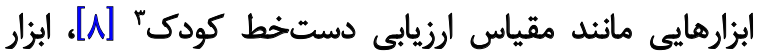


تكليف"ا: به اندازهكيرى ميزان تطابقات و كمكهايى كه هنكام

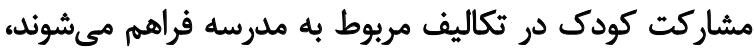

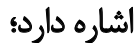

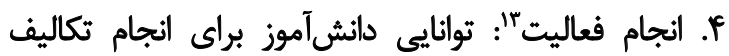

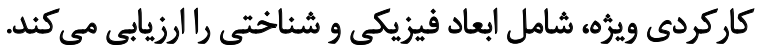

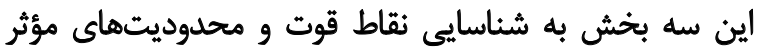

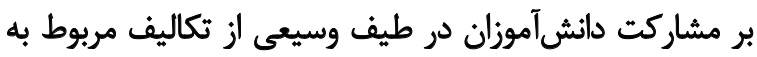

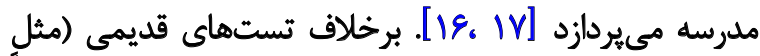

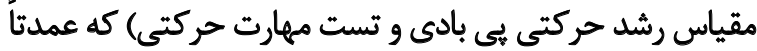

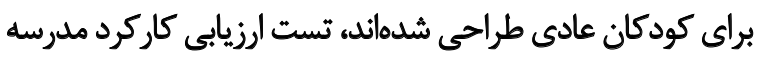

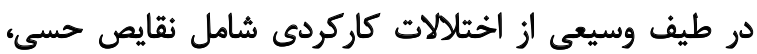

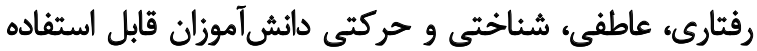

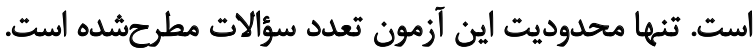

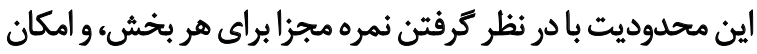
كزارش تنها يك بخش از ارزيابى تا حدودي مردي مرتفع شده است. در ايران اغلب ابزارهاى ارزيابى مورداستفاده، به سنجش

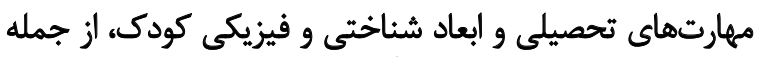

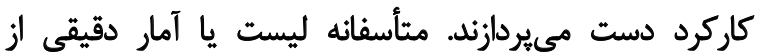

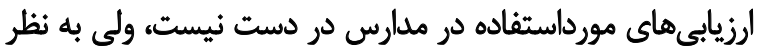

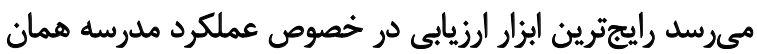

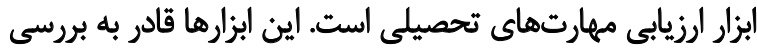

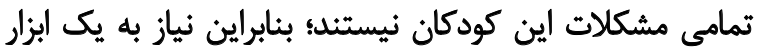

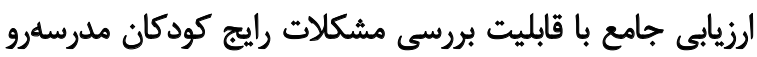

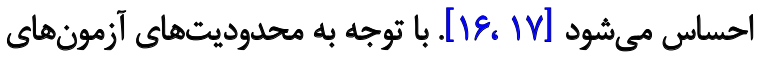

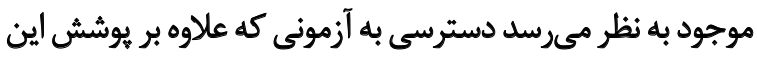

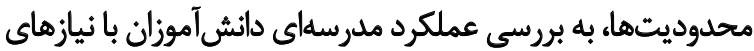

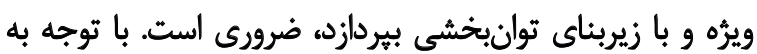

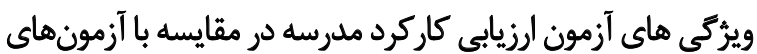
موجود در ايران و خارج از كشور، هدف از ائن مطالعه برائ برديى روائي

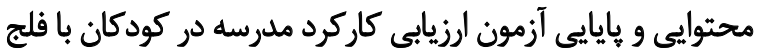

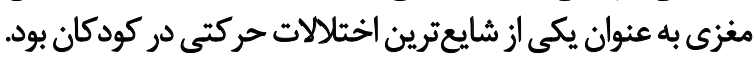

$$
\text { ووش بروسى }
$$

"يروهش حاضر يك مطالعه غيرتجربى مقطعى" (توصيفى

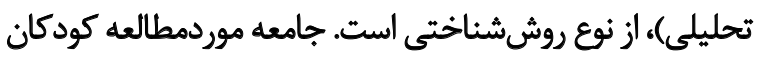

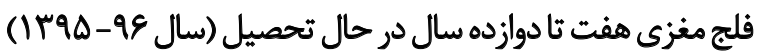

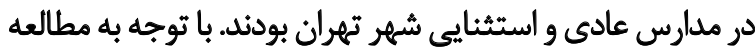

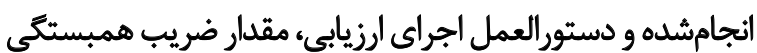

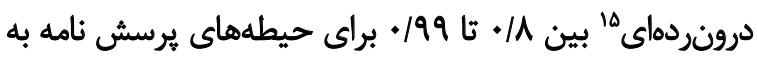

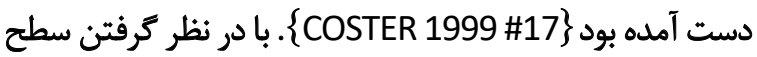

\section{3 Task Support}

13. Activity Performance

14. Cross-sectional

15. Intra-class correlatin (ICC)

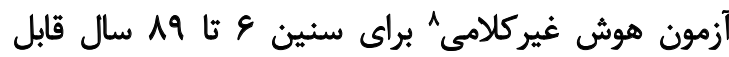

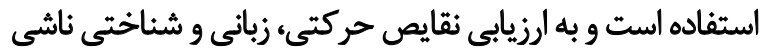

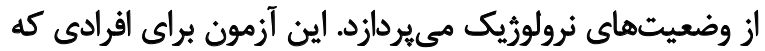

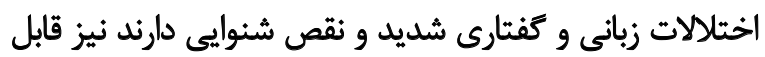

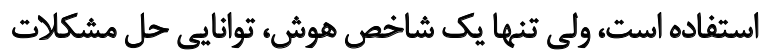
انتزاعى، را اندازهكيرى مى كند [هادئ.

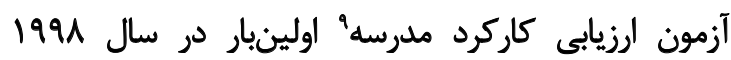

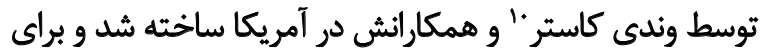

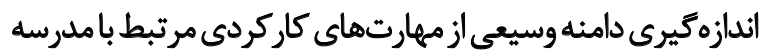

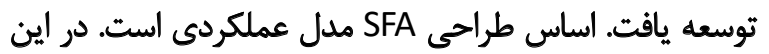

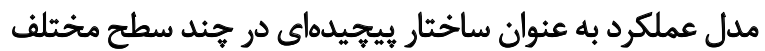

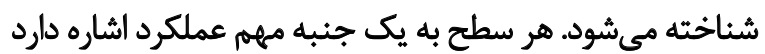

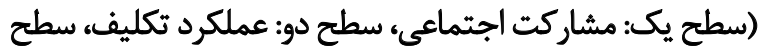

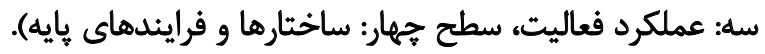

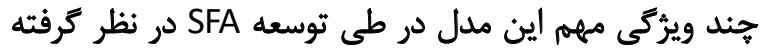

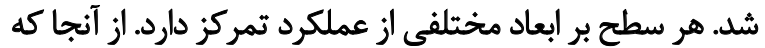

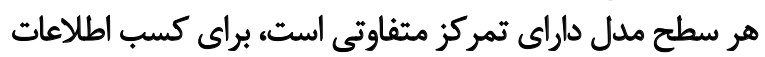

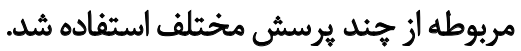

دانش فعلى در مورد رابطه بين انجام فعاليتهاي مجزا مانيد

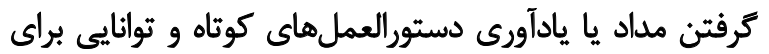

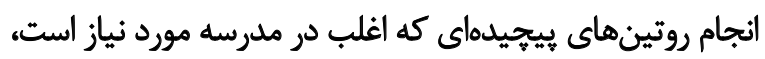

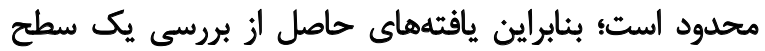

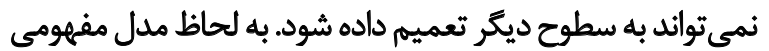

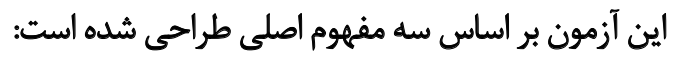
1. عملكرد كارى توسط فاكتورهاي محيطى و فردى منعكس

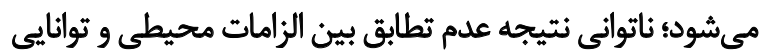

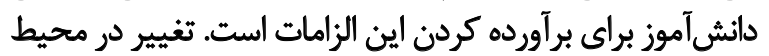

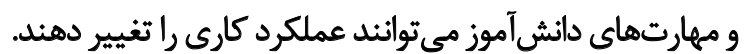
r. عملكرد كارى وابسته به بافتار است: عملكرد دانشآموز

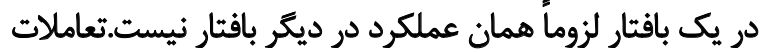

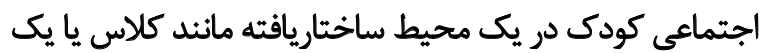

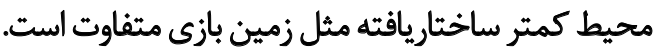

rا. عملكرد به وسيله نتيجه معين مي تيود، نه روشهاي

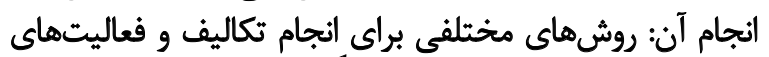

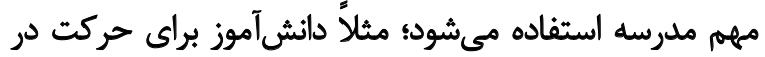

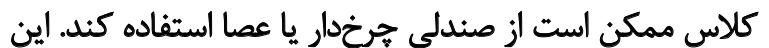

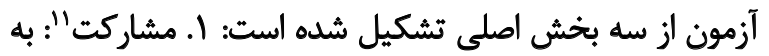

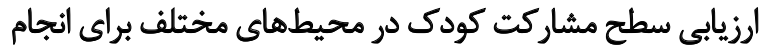

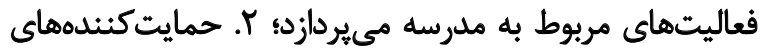

11. Participation 
كاركردى ويرهه مائند ابعاد فيزيكى و شناختى رفتارى ارزيابى

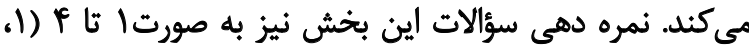

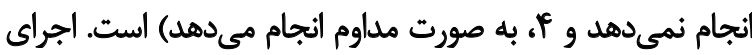

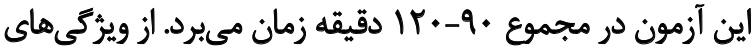

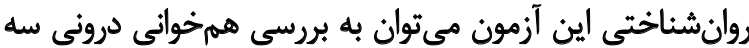

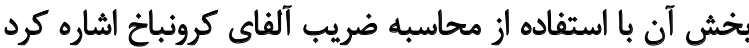

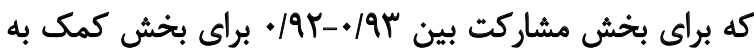

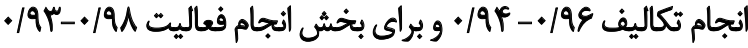

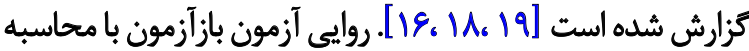

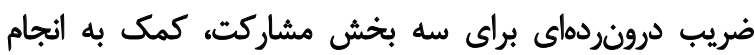

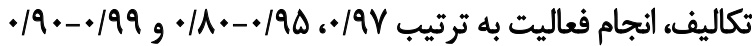

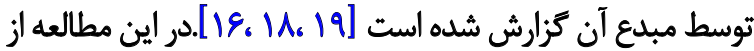

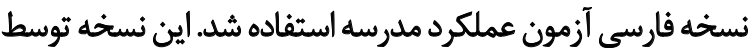

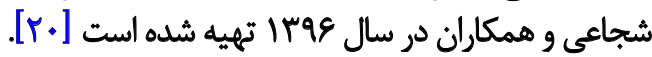

فرايند اجرا

يس از كسب مجوزهاى لازمه براي بررسى إياييى و روايى نسخه

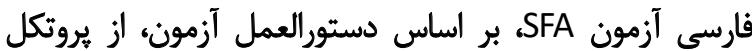

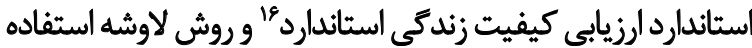

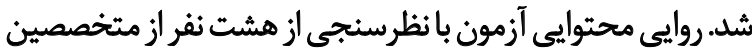

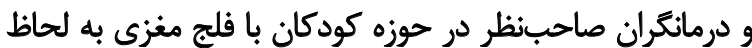

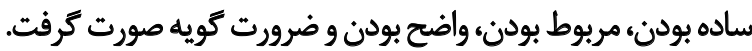

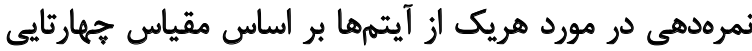

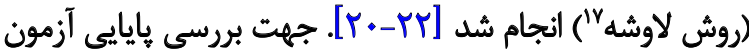

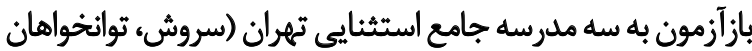

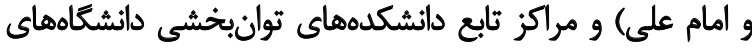

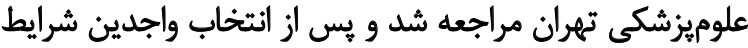

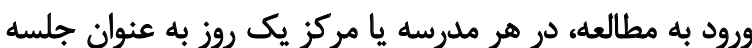
توجيهى براي شركت كنندكان ترتيب داده شدا يدا

به علت تعدد سؤالات و احتمال بروز خستخى يا كسالت حين

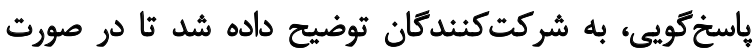

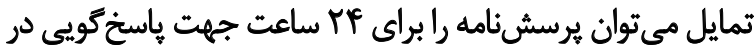

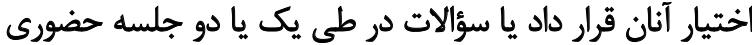

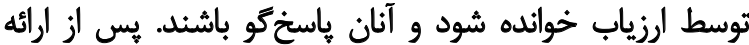

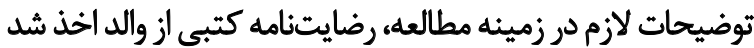

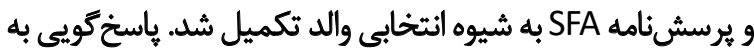

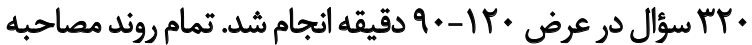

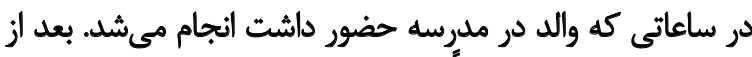

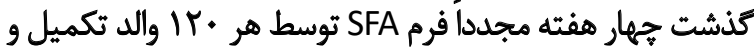

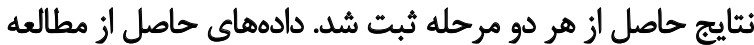

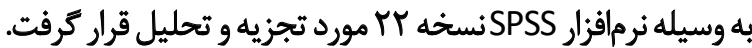

16. International Quality of Life Assessment (IQOLA) 17. Lawash

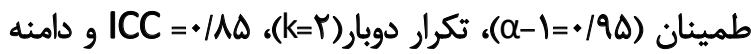

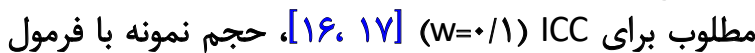

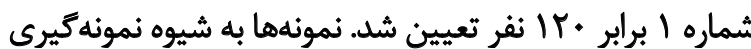
غيراحتمالى در دسترس انتخاب شدند (فرمول شماره ()).

$$
n=\frac{8 z^{2}{ }_{\alpha 2}\left(1-I C C^{2}\right)(1+(k-1) I C C)^{2}}{k(k-1) w^{2}}+1
$$

ICC $=0.85$

$\mathrm{K}=2$

\section{$W=0.1$}

$\alpha=0.05$

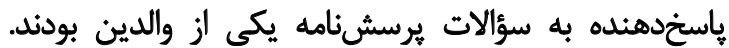

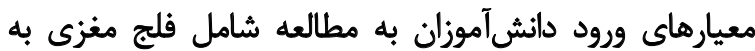
تشخيص متخصص مغز و اعصاب،سن بين هفت تأ دوازده سال، فلج مغزى بدون معلوليت همراه (نابينايى يا ناشنوايى) و وتكميل

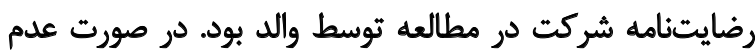

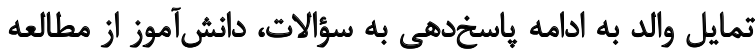
خارج مىشد.

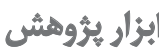
در اين مطالعه از دو برسشنامه استفاده شد: الف) يرسشنامه ويرّكى هاى فردى كه شامل اطلاعات

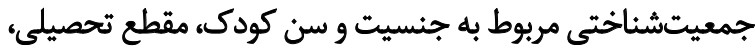
نوع فلج مغزى دانش آموزان بود و توسط يكى ازيت والدين تكميل مي كيد. ب) آزمون عملكرد مدرسه: آزمون عملكرد مدرسه اولينبار

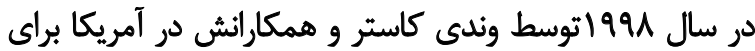

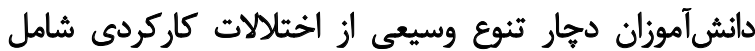

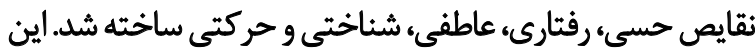

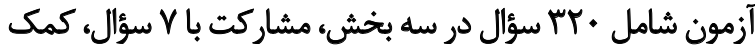

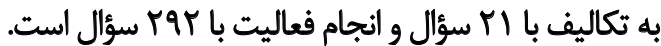
حوزه مشاركت، به ارزيابى سطح مشاركت كودك در محيطهاي

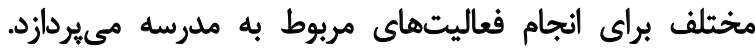

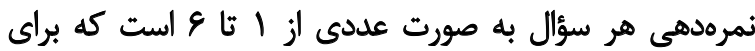

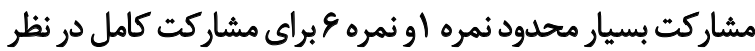

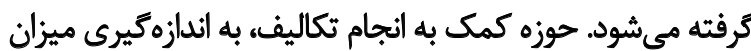

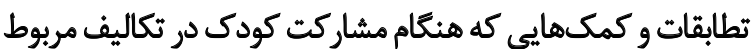

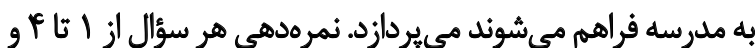

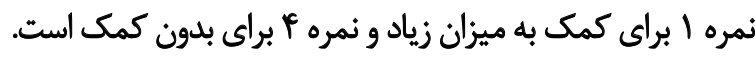
حوزه انجام فعاليت، ثوانايى دانشآموز را براى انجام تكاليف 
خوب تا عالى بود كه نشان مي دهد با توجه به تعاريف، همخوانى

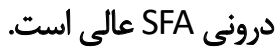

جهت بررسى همسانى باياييى آزمون بازآزمون از محاسبه

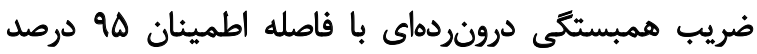

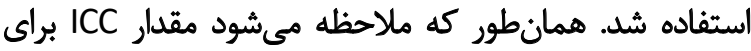

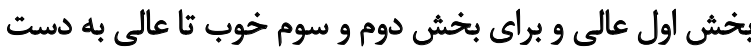
آمده است. نتايج در جدول شماره بر بش نشان داده شده است.

بحث

بسيارى از ابزارهاى ارزيابى موجود بر اساس مهارتهاى دراي

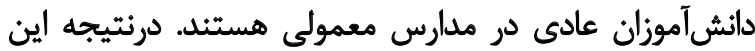

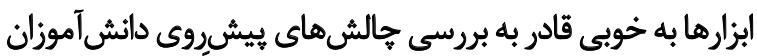

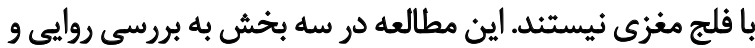

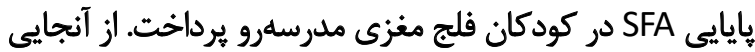

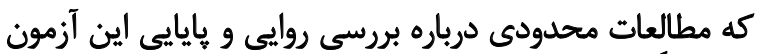

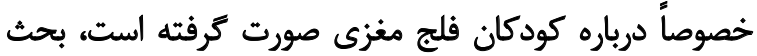
و مقايسه نتايج اين مطالعه با مطالعات قبلى فيلى با دشوارى همراه

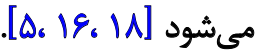

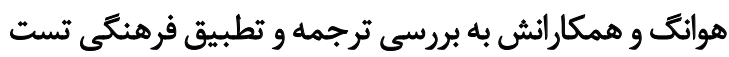

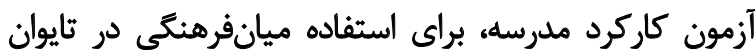

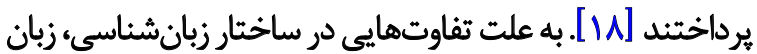

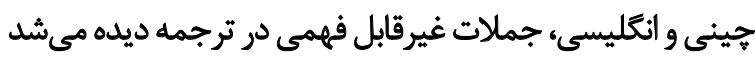

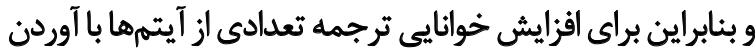

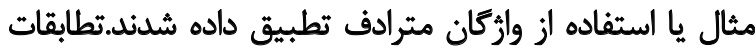

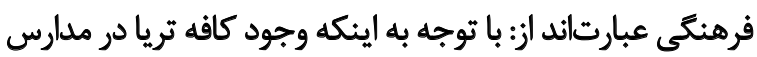

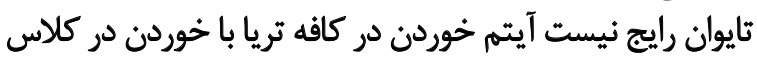

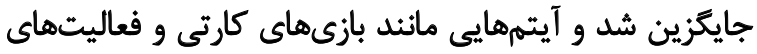

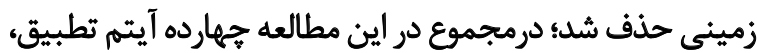

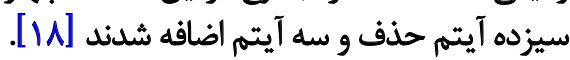

در مطالعه شجاعى و همكارانش در ايران تعداى از آيتمها

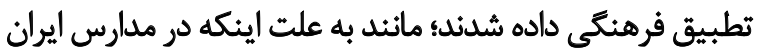

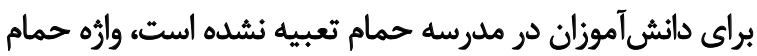

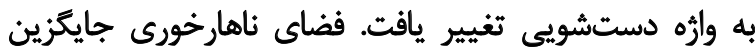

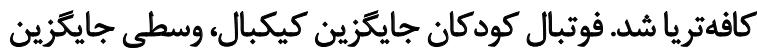

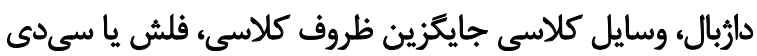

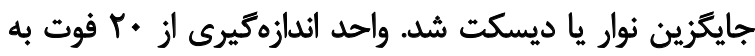

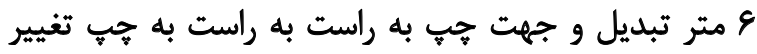

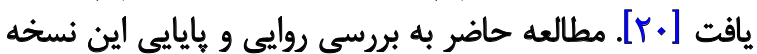
فارسى شافه يرداخته اسطت

نتايج مطالعه حاضر نشان داد تمام آيتمهاي نسخه فارسى آنى

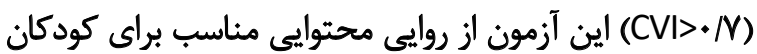

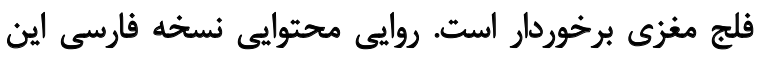

تئزيه و تجليل آمارى

براى توصيف متغيرها با روش آمارى توصيفى به محاسبه

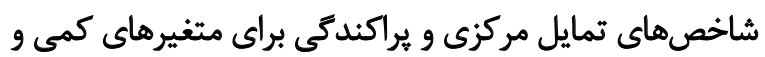

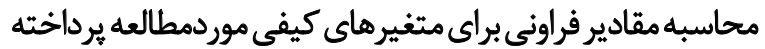

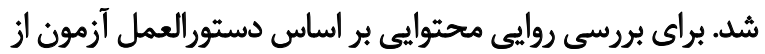

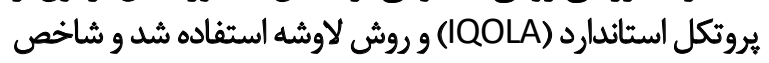

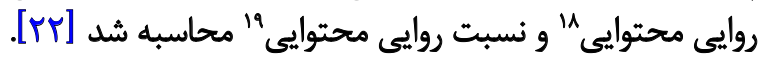

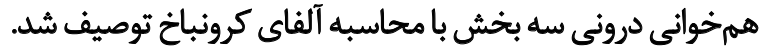

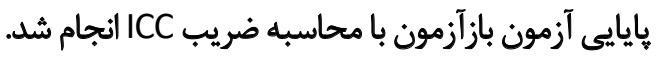

ياقتهاهـا

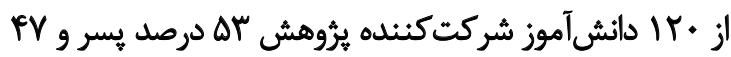

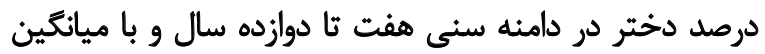

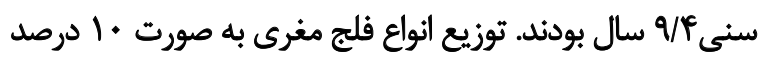

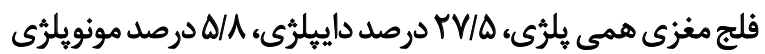

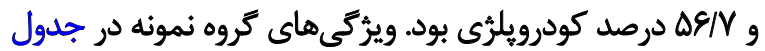
شماره ا ارائه شده است كودرئ.

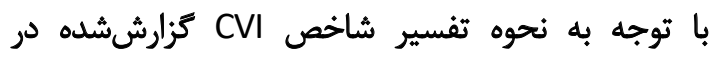

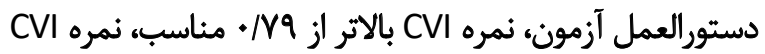

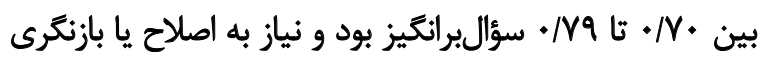

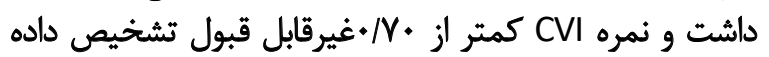

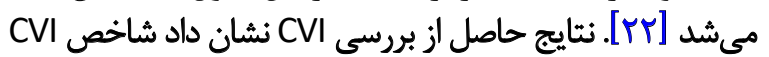

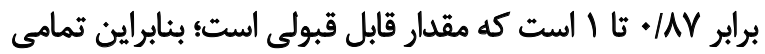
آيتم ها از روايى محتوايى مناسبى برخوردار هستئد

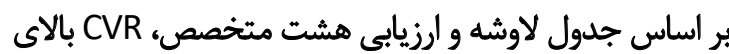

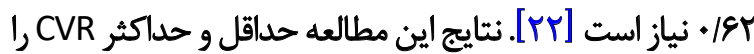

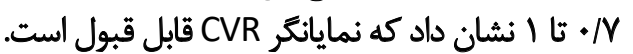

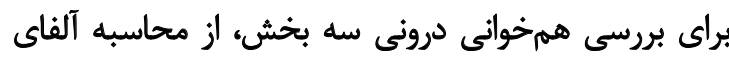

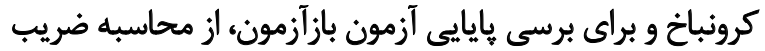

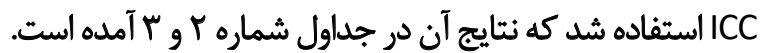

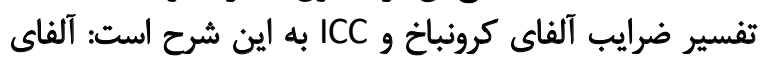

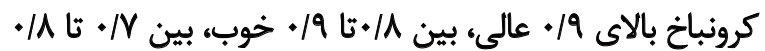

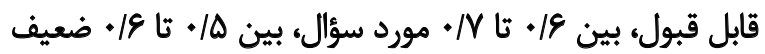

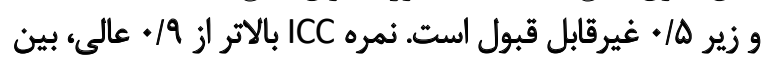

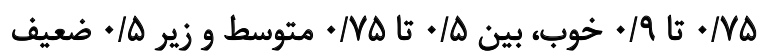

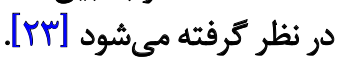

جدول شماره Y نتايج بررسى همسانى درونى با محاسبه

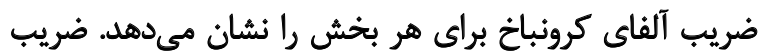

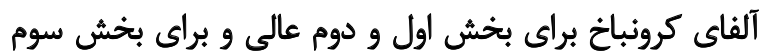

18. Content Validity Index (CVI)

19. Content Validity Ratio (CVR) 
جدول ا. مشخصات فردى كودكان و والدين شركت كنينده

\begin{tabular}{|c|c|c|}
\hline \multicolumn{2}{|c|}{ فراوانى (درصد) } & \multirow[t]{2}{*}{ نام متغير } \\
\hline$K E(T / M)$ & 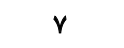 & \\
\hline $\mathscr{V}(1 \% / \mu)$ & $\lambda$ & \multirow{5}{*}{ (بر حسب سال) } \\
\hline$I \gamma(\mid r / T)$ & 9 & \\
\hline$M(N M)$ & 1. & \\
\hline$M(N Y)$ & 11 & \\
\hline $\operatorname{Ir}(I F / T)$ & r & \\
\hline$r(\Delta / \Lambda)$ & مونويلثَى & \multirow{5}{*}{ نوع فلج مغزى } \\
\hline$M(1)$. & همى بيلثى & \\
\hline & & \\
\hline$\pi r(T V / \Delta)$ & إييلزى & \\
\hline $\operatorname{sA}(\Delta S / M)$ & كوادريلرى & \\
\hline$r \cdot(1 .)$. & & كل \\
\hline$\Delta \&(\Leftrightarrow \& /)$ & دختر & \multirow{3}{*}{ جنسيت كودكان } \\
\hline & & \\
\hline$\phi P(\Delta r / T)$ & يسر & \\
\hline$r \cdot(1.0)$ & & كل \\
\hline एq $(T / \Delta)$ & سيكل & \multirow{4}{*}{ سراقيين تحصيلات } \\
\hline$P(T N M)$ & دييلم & \\
\hline$r \Delta(T \cdot / \Lambda)$ & كارشناسى & \\
\hline $\left.1 \cdot(N)^{\prime}\right)$ & ارشد و بالاتر & \\
\hline$r \cdot(1 . .0)$ & & كل \\
\hline $\operatorname{ar}(\Lambda \cdot / \Lambda)$ & زن & \multirow{2}{*}{ مراقييين } \\
\hline$r(19 / 4)$ & مرد & \\
\hline$r \cdot(1 \cdot)$. & & كل \\
\hline rA $(r M)$ & $r_{*}-r_{*}$ & \multirow{4}{*}{ (ير حسب سرالي) } \\
\hline$\Delta \rho(\varphi / M)$ & $r \cdot-\varphi$. & \\
\hline$M(10)$ & $f+-\Delta$. & \\
\hline$A(\varepsilon / V)$ & $0 .-8$. & \\
\hline$r \cdot(1.0)$ & & كل \\
\hline
\end{tabular}


جدول ب. نتايج بررسى همسائى درونى يرسش نامه آزمون عملكرد مدرسه به روش محاسبه آلفاى كروئباخ

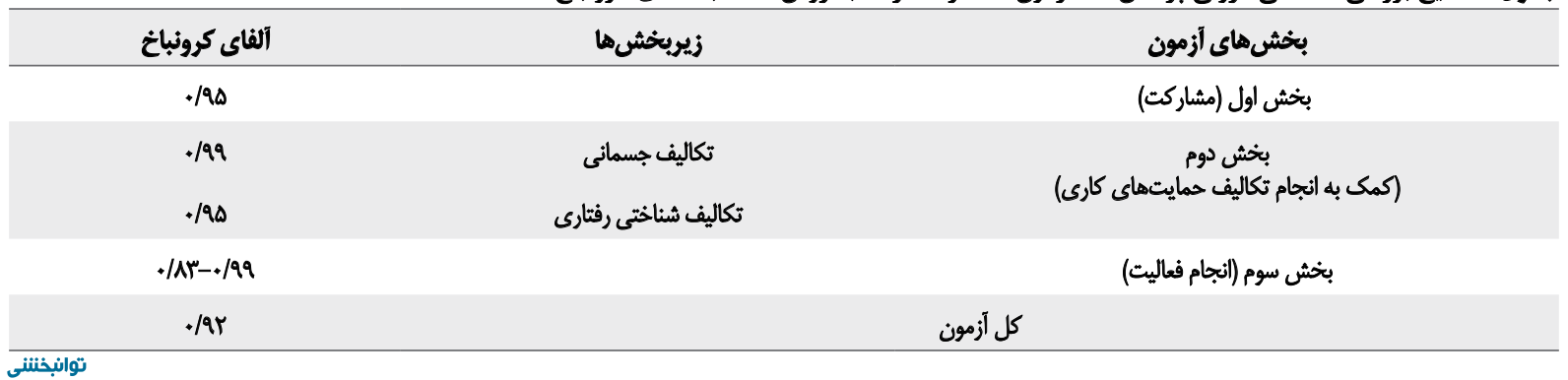

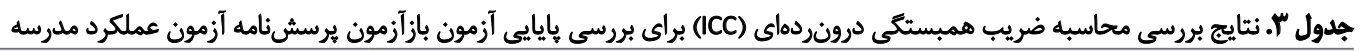

\begin{tabular}{|c|c|c|}
\hline همبستيى درونخوشهاى & زيريخشها & بحش هاي أزمون \\
\hline.$/ 9$. & & بخش اول (مشاركت) \\
\hline & ت كاليف جسمانى & (كمك به انجام تكاليف حمايتش دواى كارى) \\
\hline - /Ar & تكاليف شناختى رفتارى & \\
\hline -/Af-./QY & & بخش سوم ( انجام فعاليت) \\
\hline .119 & & كل أزمون \\
\hline
\end{tabular}

بررسى روايى محتوايى روى · Tr والد دانشآموز فلج مغزى انجام شد و در كل روايى محتوايى مناسبى بله دست مست آمد.

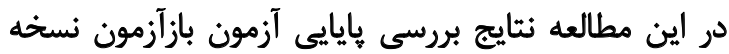

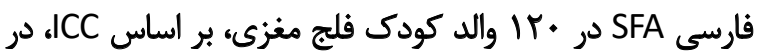

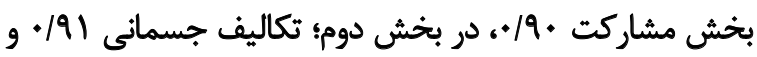

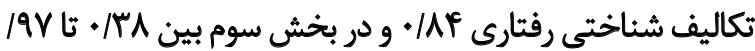

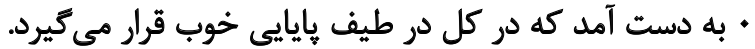

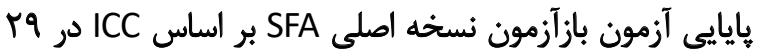

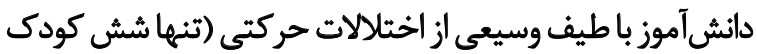

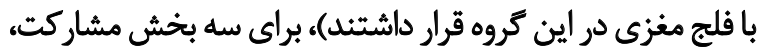

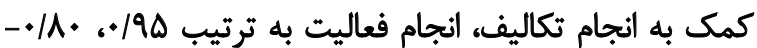

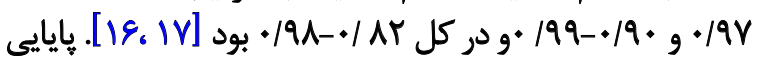
آزمون بازآزمون نسخه جيني SFA در جهمارده كودك فلج مغزي

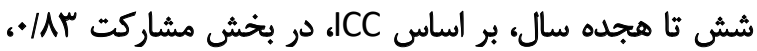

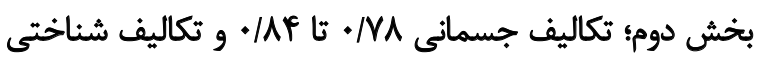

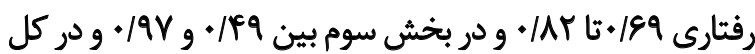
متوسط كزارش شد [rه].

بايايي آزمون بازآزمون نسخه فارسي SFA توسط جهل

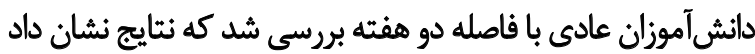

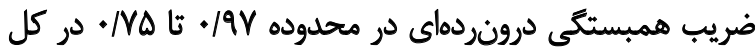

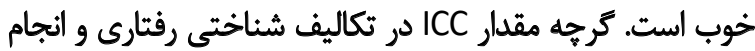

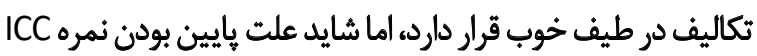

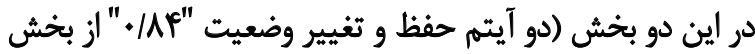

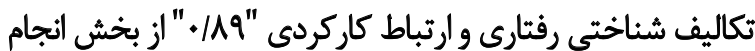

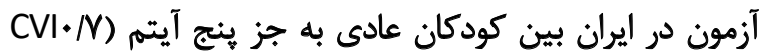

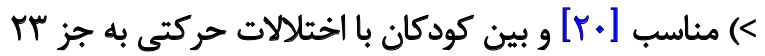

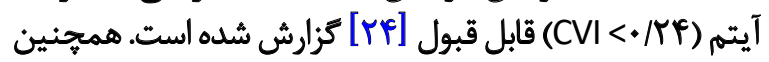

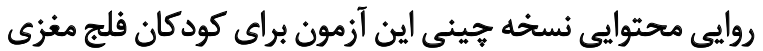
مناسب (CVI>/V) بوده است [T/V)]

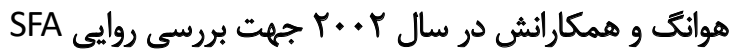

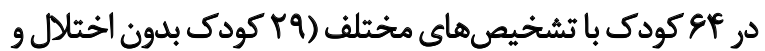

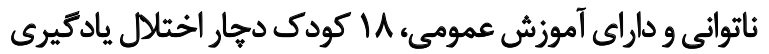

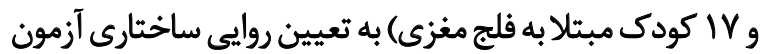

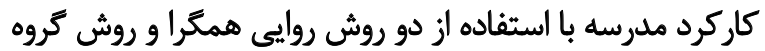

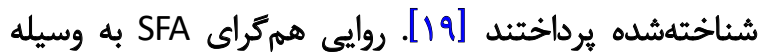

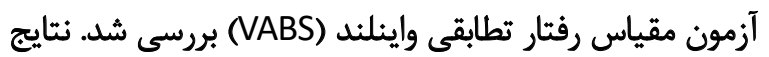

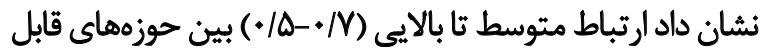

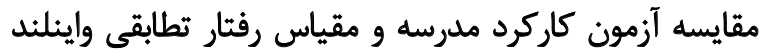

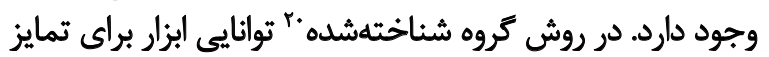

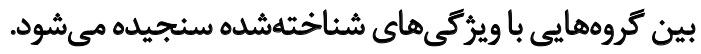

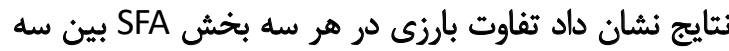

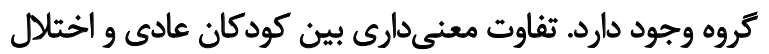

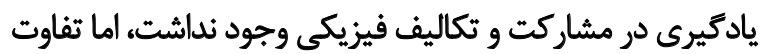

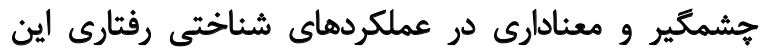

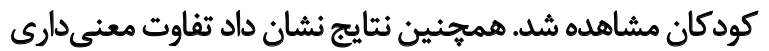

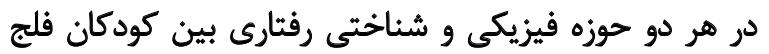
مغزى و كودكان عادى وجود داشت [9 1]]. اين مطالعه فقط بـ به فيه

20. Known groups method 
ميىتوان از آن به صورت يرؤهشى و بالينى براي ارزيابى كاركرد مدرسه كودكان فلج مغزى استفاده كرد.

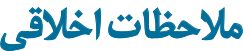

\section{بيروى أز اصول اخلاق يُوهش}

IR.USWR. براى اجراى اين مطالعه ابتدا كد اخلاقى به شماره

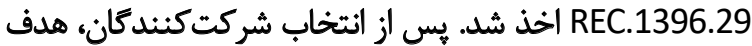

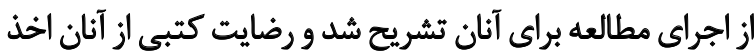

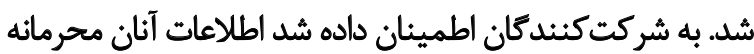

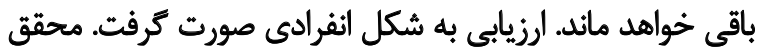

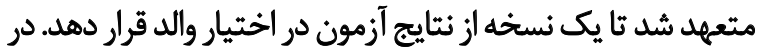

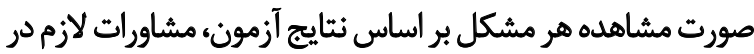

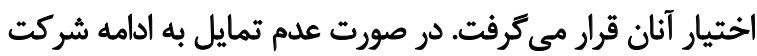

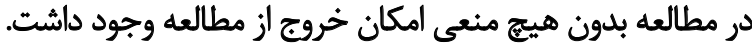
اين مطالعه هيج ضرر يا هزينهاي براى والد و دانش آموز نداشت مطان.

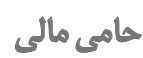

اين مطالعه از ياياننامه كارشناسىارشد نويسنده اول كروه

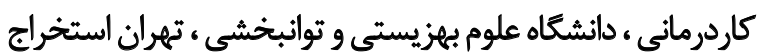

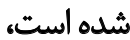

\section{مشاركت نويسندنًان}

مفهومسازى: نازيلا اكبر فهيمي، مهلى رصافيانى؛ روشي

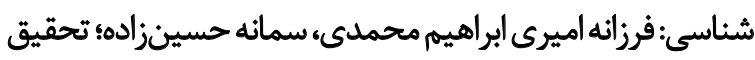

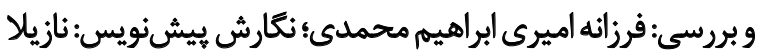

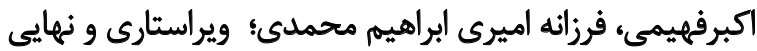

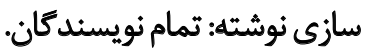

$$
\text { تقارض مثافع }
$$

بنابر اظهار نويسندكان اين مقاله تعارض منافع ندارد.

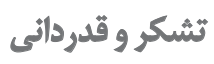

نويسندكان بر خود واجب مى انيند از همكارى ارزشمئد

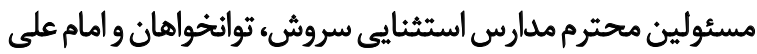

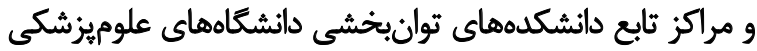

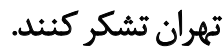

دكاليف) رابتوان به دركيرى حركتى وسيع (كوادرو يلرّى بودن

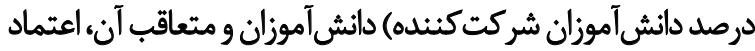
به نفس هايين در حركت و برقرارى ارتباط مربوط دانست. نتايج بررسى همسانى درونى نسخه فارسى SFA در • با والد

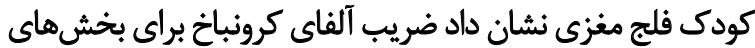

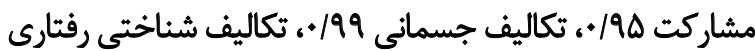

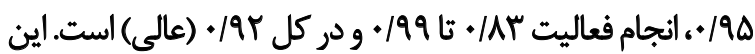

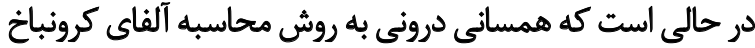

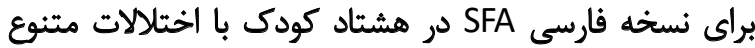

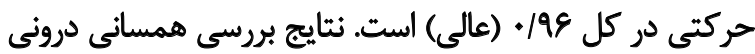

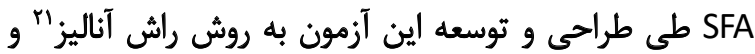

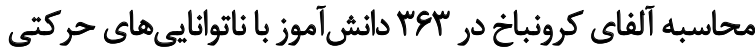

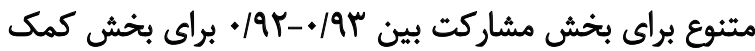

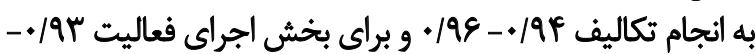

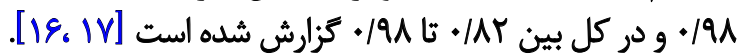
در نسخه جينى نيز آلفاى كرونباخ براى باه دانشآموز فلج مغزى تئي

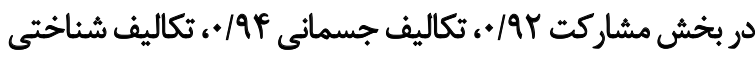

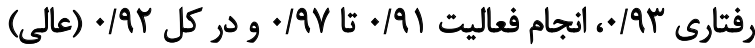

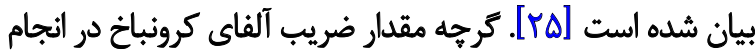

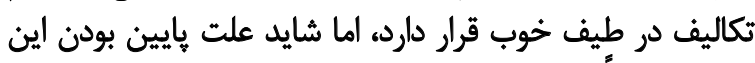

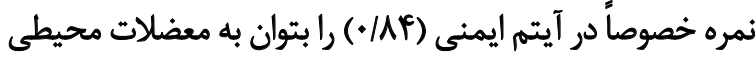

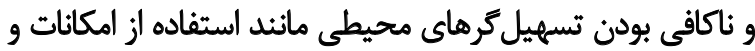
تجهيزات بهتر آموزشى در مدارس استثنايى تهران مران مربوط دانست استفاده المانات

در بايان مي توان نتيجه كيرى كرد نسخه فارسى آزمون عملكرد

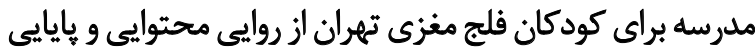

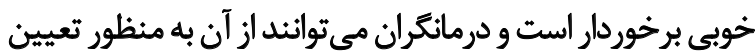

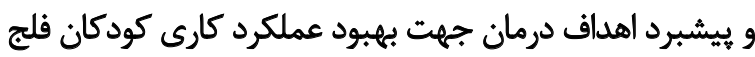

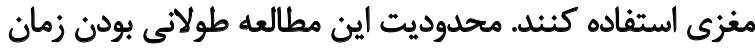

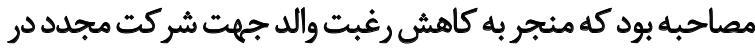

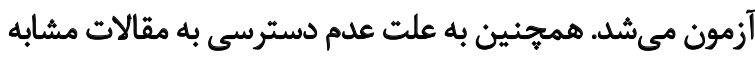

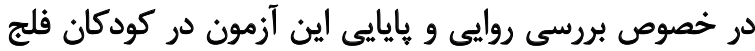

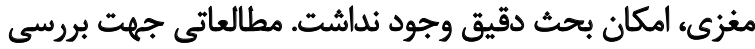

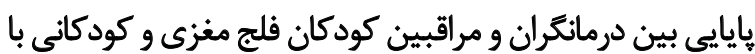

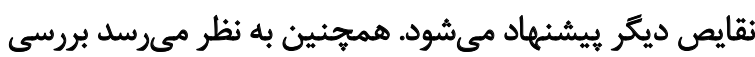

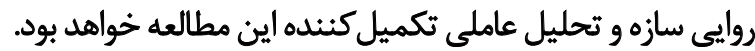

$$
\text { تيجيديرى }
$$

نتايج نشان داد نسخه فارسى آزمون عملكرد مدرسه از روايى

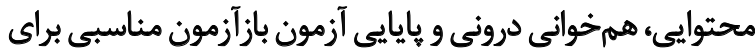

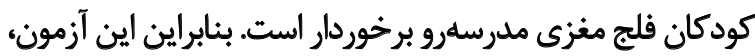

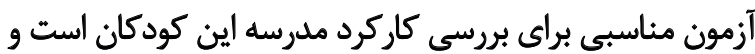

r). Rash analysis 


\section{References}

[1] AkbarFahimi N, Rassafiani M, Soleimani F, Vameghi R, Kazemnejad A, Nobakht Z. [Validity and reliability of Farsi version of cerebral palsy-quality of life questionnaire (Persian)]. Journal of Rehabilitation. 2013; 13(5):73-83. http://rehabilitationj.uswr. ac.ir/browse.php?a_code $=\mathrm{A}-10-543-2 \& \mathrm{ftxt}=1$

[2] Case-Smith J, O'Brien JC. Occupational therapy for children and adolescents-e-book. Berlin: Elsevier Health Sciences; 2014. https://books.google.com/books?id=0-DsBAAAQBAJ\&prints $\mathrm{ec}=$ frontcover $\& \mathrm{dq}=$

[3] Abdollahipour F, Alizadeh Zarei M, Akbar Fahimi M, Karamali Esmaeili S. [Study of face and content validity of the Persian version of behavior rating inventory of executive function, preschool version (Persian)]. Archives of Rehabilitation. 2016; 17(1):12-9. [DOI:10.20286/jrehab-170110]

[4] Pratt B, Baker KW, Gaebler-Spira DJ. Participation of the child with cerebral palsy in the home, school, and community: A review of the literature. Journal of Pediatric Rehabilitation Medicine. 2008; 1(2):101-11. https://content.iospress.com/articles/ journal-of-pediatric-rehabilitation-medicine/prm00022

[5] Egilson ST, Coster WJ. School function assessment: Performance of Icelandic students with special needs. Scandinavian Journal of Occupational Therapy. 2004; 11(4):163-70. [DOI:10.1080/11038120410020737]

[6] Bedell GM, Khetani MA, Cousins MA, Coster WJ, Law MC. Parent perspectives to inform development of measures of children's participation and environment. Archives of Physical Medicine and Rehabilitation. 2011; 92(5):765-73. [DOI:10.1016/j. apmr.2010.12.029] [PMID]

[7] King GA, Law M, King S, Hurley P, Hanna S, Kertoy M, Rosenbaum P. Measuring children's participation in recreation and leisure activities: Construct validation of the CAPE and PAC. Child: Care, Health and Development. 2007; 33(1):28-39. [DOI: 10.1111/j.1365-2214.2006.00613.x] [PMID]

[8] Rosenblum S, Weiss PL, Parush S. Product and process evaluation of handwriting difficulties. Educational Psychology Review. 2003; 15(1):41-81. [DOI:10.1023/A:1021371425220]

[9] Koziatek SM, Powell NJ. A validity study of the Evaluation Tool of Children's Handwriting-Cursive. American Journal of Occupational Therapy. 2002; 56(4):446-53. [DOI:10.5014/ ajot.56.4.446] [PMID]

[10] Reisman JE. Development and reliability of the research version of the Minnesota Handwriting Test. Physical \& Occupational Therapy in Pediatrics. 1993; 13(2):41-55. [DOI:10.1080/ J006v13n02_03]

[11] Feder K, \& Majnemer A. Children's handwriting evaluation tools and their psychometric properties. Physical \& Occupational Therapy in Pediatrics. 2003: 23(3):65-84. [DOI:10.1080/ J006v23n03_05]

[12] Gandek B, Ware Jr JE. Methods for validating and norming translations of health status questionnaires: The IQOLA project approach. Journal of Clinical Epidemiology. 1998; 51(11):953-9. [DOI:10.1016/S0895-4356(98)00086-9]
[13] Groth-Marnat G. Handbook of psychological assessment. Hoboken: John Wiley \& Sons; 2009. http://al-edu.com/wpcontent/uploads/2014/05/Groth-Marnat-The-Handbook-ofPsychological-Assessment.pdf

[14] Wechsler D. Wechsler Preschool and Primary Scale of intelligence-fourth edition. London: Pearson; 2012. [DOI:10.1037/ t15171-000]

[15] Hammill DD, Pearson N. Comprehensive Test of Nonverbal Intelligence; Handbook of nonverbal assessment. Springer; 2017. [DOI:10.1007/978-3-319-50604-3_10]

[16] Coster W, Deeney T, Haltiwanger J, Halley S. School function assessment user's manual. Boston: Sargent College of Health and RehabilitationScience; 1998.

[17] Coster WJ, Mancini MC, Ludlow LH. Factor structure of the school function assessment. Educational and Psychological Measurement.1999;59(4):665-77. [DOI:10.1177/00131649921970099]

[18] Hwang JL, Nochajski SM, Linn RT, Wu YWB. The development of the School Function Assessment-Chinese version for cross-cultural use in Taiwan. Occupational Therapy International. 2004; 11(1):26-39. [DOI:10.1002/oti.195] [PMID]

[19] Hwang JL, Davies PL, Taylor MP, Gavin WJ. Validation of school function assessment with elementary school children. OTJR: Occupation, Participation and Health. 2002; 22(2):48-58. [DOI:10.1177/153944920202200202]

[20] Shojaee M, Alizadeh Zarei M, Hasani Mehraban A. Translation, face and content validity of the Persian version of school function assessment. Middle East Journal of Rehabilitation and Health Studies. 2017; 4(4):e14594. [DOI:10.5812/mejrh.14594]

[21] Chehelamirani N, Sahaf R, Rassafiani M, Bakhshi E. [Validity and reliability of WHOQOL-DIS Questionnaire in Iranian older people with disability (Persian)]. Archives of Rehabilitation. 2016; 16(4):334-45. http://rehabilitationj.uswr.ac.ir/article-1-1708-fa. html

[22] Lawshe C. A quantitative approach to content validity. Personnel Psychology. 1975; 28(4):563-75. [DOI:10.1111/j.1744-6570.1975. tb01393.x]

[23] Koo TK, Li MY. A guideline of selecting and reporting intraclass correlation coefficients for reliability research. Journal of Chiropractic Medicine. 2016; 15(2):155-63. [DOI:10.1016/j. jcm.2016.02.012] [PMID] [PMCID]

[24] Kouhbanani NG, Zarei MA, Lajevardi L, Rahimzadegan H, Shojaei A. The reliability of the Persian version of the school function assessment in iranian students 6 to 12 years old in Tehran's schools. Middle East Journal of Rehabilitation and Health. 2018; 5(1):e64364. [DOI:10.5812/mejrh.64364]

[25] Li XL, Dong VAQ, Fong KN. Reliability and validity of school function assessment for children with cerebral palsy in Guangzhou, China. Hong Kong Journal of Occupational Therapy. 2015; 26(1):43-50. [DOI:10.1016/j.hkjot.2015.12.001] 